\title{
Carga horária de saúde coletiva antes do internato em escolas médicas brasileiras
}

\author{
Community Health course load before clerkship in Brazilian medical schools
}

\author{
Sofia Romay Oliveira' (1) | sromay10@gmail.com \\ Athos Paulo Santos Martini' (D) athosmartini@gmail.com \\ Suely Grosseman ${ }^{1,2}$ (D) sgrosseman@gmail.com
}

\section{RESUMO}

Introdução: Os limites da carga horária (CH) de saúde coletiva (SC) antes do internato (Al) não estão definidos nas Diretrizes Curriculares Nacionais, nem em outros documentos, e não foram investigados de forma abrangente em estudos nacionais.

Objetivo: Analisar a CH de SC Al em escolas médicas (EM) brasileiras.

Método: Estudo transversal, com escolas reconhecidas pelo Ministério da Educação que iniciaram as atividades até 31 de dezembro de 2017. Os sites das 323 escolas existentes foram consultados, e foram incluídas aquelas que disponibilizavam seus currículos na internet com detalhamento de $\mathrm{CH}$ de SC Al. As variáveis foram região geográfica e administração da escola, $\mathrm{CH}$ do curso de medicina e $\mathrm{CH}$ de SC Al, incluindo também as $\mathrm{CH}$ de epidemiologia, bioestatística e saúde do trabalhador. Os dados foram analisados usando-se estatística descritiva, teste de qui-quadrado $\left(X^{2}\right)$ de Pearson para variáveis categóricas e testes Mann-Whitney-U $(U)$ e X² de Kruskal-Wallis para variáveis contínuas.

Resultados: Foram incluídas 222 das 323 EM existentes (68,7\%), sendo 83 gratuitas (37,4\%) e 139 não gratuitas (62,6\%). A mediana da $\mathrm{CH}$ total de SC Al foi de 440,0 horas $\left(P_{25-75}=300,0-640,0\right)$, equivalente a 5,4\% $\left(P_{25-75}=3,5-7,8\right)$ da CH total do curso. A mediana da CH de SC Al em horas das escolas privadas e municipais foi de, respectivamente, 480,0 $\left(P_{25-75}=330,7-679,2\right)$ e $576,0\left(P_{25-75}=360,0\right.$ - 766,0); no caso das estaduais e federais, a mediana foi de, respectivamente, $337,0\left(P_{25-75}=281,2-524,2\right)$ e $370,0\left(P_{25-75}=300,0-480,0\right), X^{2}(3)=11,48, p=0,009$. As escolas não gratuitas tiveram mediana de $\mathrm{CH}$ total de SC de 500,0 horas $\left(\mathrm{P}_{25-75}=336,0-690,0\right)$ e as gratuitas de 364,0 horas $\left(P_{25-75}=285,0-504,0\right), \mathrm{U}=$ $4.259,0, z=-3,26, p=0,001$. A mediana da $C H$, em horas, de epidemiologia e bioestatística Al entre 124 escolas foi de $88,0\left(P_{25-75}=60,0-120,0\right)$ e de saúde do trabalhador entre 63 foi de $40,0\left(P_{25-75}=33,0-60,0\right)$.

Conclusões: A CH de SC Al apresenta grande variação, sendo maior em escolas não gratuitas.

Palavras-chave: Educação Médica; Currículo; Saúde Pública; Carga Horária; Saúde Coletiva.

\section{ABSTRACT}

Introduction: The limits of the Community Health $(\mathrm{CH})$ course load (CL) before medical clerkship (MC) in undergraduate medical courses are not defined neither in the National Curriculum Guidelines nor in other documents, and have not been investigated in a comprehensive way in national studies.

Objective: To analyze the community health course load before clerkship in Brazilian medical schools.

Method: Cross-sectional study of schools acknowledged by the Ministry of Education, which started activities until December 31, 2017. The sites of the 323 existing schools were accessed and the schools that had their curricula available in the internet with details of the CHCL before MC were included. The variables were school geographic region and administration type, medical course CL and CHCL before MC, also including epidemiology, biostatistics and occupational health course loads. Data were analyzed using descriptive statistics, Pearson chi-square $\left(X^{2}\right)$ for categorical variables and Mann-Whitney-U (U) and Kruskal Wallis X² tests for continuous variables.

Results: Out of 323 existing medical schools, 222 were included (68.7\%). Among the included schools, 83 were tuition-free (37.4\%) and 139 were not (62.6\%). The median of the CH CL before MC was 440.0 hours $\left(P_{25.75}=300.0-640.0\right)$, equivalent to $5.4 \%\left(P_{25-75}=3.5-7.8\right)$ of the medical course CL. The median of the $C H C L$ before $M C$ in hours in private and municipal schools was, respectively, $480.0\left(P_{25-75}=330.7-679.2\right)$ and 576.0 $\left(P_{25-75}=360.0-766.0\right)$, and of state and federal schools, respectively, $337.0\left(P_{25-75}=281.2-524.2\right)$ and $370.0\left(P_{25-75}=300.0-480.0\right), X 2(3)=11.48, p=.009$. The median of the total CHCL in non-tuition-free schools was 500.0 hours ( $\left.P_{25.75}=336.0-690.0\right)$ and in the tuition-free schools, it was 364.0 hours $\left(P_{25-75}=285.0-504.0\right), U=$ 4.259.0, $z=-3.26, p=.001$. The median of epidemiology and biostatistics $C L$ in hours before MC in the 124 schools was $88.0\left(P_{25-75}=60.0-120.0\right)$, whereas the occupational health $C L$ in 63 schools was $40.0\left(P_{25-75}=33.0-60.0\right)$.

Conclusions: The CH CL before clerkship varies widely, being higher in non-tuition-free medical schools.

Keywords: Medical Education; Curriculum; Public Health; Course Load; Community Health.

1 Universidade Federal de Santa Catarina, Florianópolis, Santa Catarina, Brasil.

2 Faculdades Pequeno Príncipe, Curitiba, Paraná, Brasil.

Editora-chefe: Daniela Chiesa

Editor associado: Pedro Tadao Hamamoto Filho

Recebido em 17/07/20; Aceito em 17/12/20.

Avaliado pelo processo de double blind review. 


\section{INTRODUÇÃO}

As universidades influenciam a comunidade em que estão inseridas e são influenciadas por ela. Os profissionais formados são agentes que compartilham o conhecimento gerado na educação superior com a população que recebe seu cuidado'.

A relação entre educação médica e o sistema de saúde vigente não é diferente. A relevância da determinação social no processo saúde-doença tomou força ao longo do século XX, impulsionando o estudo de Ciências Sociais na área de saúde pública². O movimento da Reforma Sanitária iniciou-se no Brasil nos primórdios da década de 1970 visando a uma política de saúde que considerasse a comunidade e o seu contexto. Esse movimento culminou no acordo entre grupos políticos, governamentais e privados para o estabelecimento de um Sistema Único de Saúde (SUS), de acesso universal, igualitário e gratuito, valorizando o cuidado coletivo como aspecto inseparável da saúde individual ${ }^{3}$, oficializado na Constituição Federal de 1988 e em regulamentações posteriores necessárias para sua implementação.

Paralelamente, desde a década de 1950, havia discussões iniciais quanto ao currículo médico e ao processo de ensino ${ }^{4}$, as quais foram intensificadas a partir dos anos 1980. Ficou clara a necessidade de profissionais que pudessem atuar de forma competente no novo sistema de saúde em estruturação, nos seus diferentes níveis de atenção. A transformação na formação ofertada pelas escolas médicas (EM) foi considerada como um dos meios mais eficazes para iniciar mudanças no futuro profissional e, portanto, em sua forma de atuação no sistema de saúde ${ }^{5}$.

A transformação na visão da saúde pública e de sua representação nos currículos de graduação em medicina também ocorreu em todos os continentes em que o ensino de saúde pública era abordado no curso de Medicina. Países como Canadá6, ${ }^{6,7}$ China $^{8}$, Espanha ${ }^{9}$, Austrália ${ }^{10}$, Reino Unido ${ }^{11,12} \mathrm{e}$ Gana $^{13}$ são alguns exemplos.

No Brasil, em 2001, foram elaboradas as Diretrizes Curriculares Nacionais (DCN) para os Cursos de Graduação em Medicina, para que a educação médica se alinhasse às necessidades do SUS e da população e proporcionasse a formação de médicos capacitados "a atuar [...] no processo de saúde-doença" nos diferentes níveis de atenção ${ }^{14}$. Essas DCN foram desenvolvidas a partir de uma ampla avaliação das EM e discussões posteriores entre educadores, profissionais de saúde e governo para tomada de decisão compartilhada ${ }^{14}$. $\mathrm{O}$ incentivo a uma formação de médico generalista, incluindo práticas em cenários de atenção primária e desenvolvimento de competências relacionadas à saúde pública destacou-se como diferencial em relação aos currículos existentes até então, que eram predominantemente voltados a especialidades médicas e com práticas principalmente nos hospitais. Após
13 anos, as DCN foram reestruturadas para que a educação médica respondesse "aos novos desafios das sociedades contemporâneas" contemplando adequadamente a atenção primária e valorizando a formação alinhada ao SUS. Nessa nova versão, reforçou-se o que já havia sido abordado nas DCN anteriores, e a formação médica foi abordada em três eixos: atenção à saúde, gestão em saúde e educação em saúde ${ }^{15}$.

As DCN estabelecem também a carga horária $(\mathrm{CH})$ mínima de 7.200 horas para os cursos de Medicina ${ }^{15}$ e as porcentagens do internato em relação à carga horária do curso e às áreas de saber consideradas essenciais. Porém, e até para maior flexibilidade das escolas no planejamento curricular, não sugerem limites de carga horária para conteúdos antes do internato. Se, por um lado, o fato de não haver limites definidos para as áreas de saber e conteúdos antes do internato representa uma possibilidade de maior liberdade para o planejamento curricular em cada escola, por outro, isso pode resultar em uma grande variação nas $\mathrm{CH}$ s de cada área, o que dependerá de escolhas de gestores e docentes. Nesse cenário, como afirma Lima-Gonçalves ${ }^{16}$, apesar da fundamental importância da $\mathrm{CH}$ na estrutura curricular, a decisão da $\mathrm{CH}$ a ser reservada para cada disciplina tem sido, muitas vezes, realizada como "moeda de troca" na construção dos currículos e pode ocasionar um tempo insuficiente ou excessivo em determinadas áreas.

Em relação ao ensino de Saúde Coletiva (SC), apesar de ambas as versões das $\mathrm{DCN}^{14,15}$ reconhecerem a importância dessa aprendizagem em cenários de prática da atenção primária, nem todas as EM conseguiram efetivá-la de forma abrangente. Ainda, como cada EM pode considerar de maneira diferente as $\mathrm{CH}$ s de teoria e prática a serem destinadas à $\mathrm{SC}$, os limites atuais da $\mathrm{CH}$ de $\mathrm{SC}$ nos currículos de Medicina precisam ser conhecidos.

O curso de Medicina da Universidade Federal de Santa Catarina (UFSC) mudou o currículo em 2003 para atender às recomendações das DCN. Em 2018, o currículo continha $\mathrm{CH}$ total de 7.670 horas, com um módulo denominado Saúde e Sociedade destinado à SC, ministrado do primeiro ao oitavo semestre do curso (antes do internato). A CH total do módulo era de 480 horas (6,2\% da $\mathrm{CH}$ total do currículo), com CH teórica total de 240 horas em SC, a qual incluía 90 horas de epidemiologia e bioestatística (EB) e 30 de saúde do trabalhador (ST), e com CH prática total de 240 horas, realizada na atenção primária, denominada interação comunitária. No internato, a CH de SC era de 736 horas. Portanto, a CH total de SC nessa universidade era de 1.094 horas, correspondendo a $14,3 \%$ da $\mathrm{CH}$ total do currículo. Visando avaliar a adequação dessa $\mathrm{CH}$, buscamos artigos sobre os limites de $\mathrm{CH}$ de SC antes internato nas EM do Brasil que propiciassem comparações com limites nacionais. Porém, não encontramos estudos nacionais 
abrangentes. Emergiu então a pergunta de pesquisa:

- Como estão os limites de $\mathrm{CH}$ de SC antes do internato nas EM brasileiras?

Para responder a essa questão, o objetivo deste estudo foi analisar a $\mathrm{CH}$ de SC antes do internato em EM brasileiras.

\section{MÉTODO}

\section{Delineamento e preceitos éticos}

Este estudo foi transversal e descritivo. O projeto de pesquisa não foi submetido ao Comitê de Ética porque os dados são secundários e de domínio público, disponíveis na internet.

\section{Universo e amostra}

O universo foi constituído por todas as 323 EM brasileiras existentes até setembro de 2018, reconhecidas pelo Ministério da Educação (MEC) e listadas na página do e-MEC, no site http:// emec.mec.gov.br ${ }^{17}$. Os sites de todas as escolas existentes foram acessados e os critérios para sua inclusão foram: ter início das atividades acadêmicas registrado no e-MEC antes de 31 de dezembro de 2017, ter matriz curricular ou projeto políticopedagógico (PPP), também denominado projeto político do curso, disponível na internet e conter informações sobre a $\mathrm{CH}$ de SC no curso.

\section{Coleta de dados}

Os dados foram coletados entre março e setembro de 2018.

Após consulta à página do e-MEC ${ }^{17}$, o website oficial de cada instituição foi acessado a partir deste site, com o propósito de obter a matriz curricular e/ou o PPP. Quando estes documentos não estavam disponíveis, foi realizada pesquisa da página da universidade na internet, via Google ${ }^{\circledR}$, usando-se a chave de busca: ("nome da universidade") AND ("medicina") AND ("matriz curricular" OR "projeto político pedagógico" OR "projeto político do curso"). Quando disponíveis, os programas de aprendizagem/planos de ensino, as ementas e os conteúdos de $\mathrm{CH}$ foram analisados para confirmar se toda a $\mathrm{CH}$ era destinada à $\mathrm{SC}$ e para diferenciar as $\mathrm{CH}$ s de teoria e prática.

Para definir módulos e disciplinas a serem incluídos como SC, foi utilizado como base o currículo de SC da UFSC: Educação em Saúde, Epidemiologia e Bioestatística, Políticas Públicas de Saúde, Medicina da Família e Comunidade, Planejamento, Gestão e Avaliação dos Serviços, Saúde do Trabalhador e Programas de Saúde. Foram incluídos também módulos, disciplinas e conteúdos relacionados à SC, cujos nomes eram diferentes dos anteriores e abordavam princípios e funcionamento do SUS, atributos da atenção primária em saúde, gestão pública, sistemas de informação em saúde, bioestatística, planejamento em saúde e saúde do trabalhador.
Esse conjunto apresenta mais conteúdos do que os incluídos por alguns autores. Oliveira et al. ${ }^{18}$, por exemplo, não incluem Estatística e Saúde do Trabalhador, apesar da importância de análises estatísticas em estudos populacionais relativos à SC e da relevância da saúde ocupacional no contexto de saúde coletiva, confirmada inclusive pela Resolução $n^{\circ}$ $1.488 / 98$ do Conselho Federal de Medicina (CFM) ${ }^{19}$, que dispõe sobre normas específicas para todos os médicos que atendam o trabalhador.

Ainda que as Ciências Sociais e Humanas fossem consideradas relevantes e essenciais para a SC, seus conteúdos não foram incluídos e contabilizados no presente estudo por serem mais frequentemente ensinados de forma separada da saúde coletiva/pública e ministrados por departamentos de outras áreas de saber, como a Antropologia e Sociologia.

As variáveis incluídas foram: região geográfica e administração da escola, $\mathrm{CH}$ total do curso de Medicina, $\mathrm{CH}$ total obrigatória de SC antes do internato, $\mathrm{CH}$ de teoria e de prática (abrangendo atividades realizadas na comunidade, como interação ensino-serviço) e as CHs de EB e ST.

Apesar de inseridas no conteúdo total de SC, as $\mathrm{CH}$ de EB e ST foram individualizadas, para maior compreensão sobre seus limites.

As CHs foram padronizadas em "hora/relógio", correspondente a uma hora real de 60 minutos. Quando a escola especificava que a hora/aula apresentada era diferente da hora/ relógio, essa hora foi calculada para o correspondente em hora real. Antes da conversão, algumas escolas apresentavam uma hora/aula correspondendo a 60, 50 ou 45 minutos reais.

\section{Análise de dados}

Os dados foram inseridos em banco de dados do software Microsoft Excel 2013.

A análise foi realizada usando-se estatística descritiva, com frequências absoluta e relativa para variáveis categóricas, e medidas de tendência central para variáveis contínuas. A normalidade da distribuição das variáveis contínuas foi analisada pelo teste Kolmogorov-Smirnov, sendo a distribuição considerada normal quando $p \geq 0,05$. As medidas de tendência central para variáveis com distribuição normal foram média e desvio padrão e as medidas para variáveis com distribuição não normal foram mediana e percentis 25 e $75\left(\mathrm{P}_{25-75}\right)$. Para propiciar comparações com outros estudos, no caso de distribuição não normal, foram também fornecidas a média com desvio padrão (DP).

Para análise de diferenças entre grupos, foi usado o teste de qui-quadrado $\left(\mathrm{X}^{2}\right)$ de Pearson para variáveis categóricas e os testes de Mann-Whitney-U (U) e $X^{2}$ de Kruskal-Wallis para variáveis contínuas.

Além da análise individualizada da administração das 
escolas, esta categoria também foi aglutinada em escolas gratuitas (escolas federais e estaduais) e não gratuitas (escolas privadas e municipais).

O nível de significância admitido foi de $p<0,05$.

\section{RESULTADOS}

A Tabela 1 apresenta a distribuição das escolas incluídas e não incluídas por região geográfica, administração e gratuidade. Como pode ser observado, 222 das 323 EM existentes foram incluídas (68,7\%). Não houve diferença entre as escolas incluídas e não incluídas, segundo a administração e gratuidade dentro de cada região. Também, o número de escolas privadas e não gratuitas é maior no Brasil do que o de gratuitas $(63,5 \%)$. As regiões Sudeste e a Sul têm a maior proporção de escolas privadas e não gratuitas em relação ao número de escolas em cada uma destas regiões (respectivamente de 77,8\% e 64,2\%). As demais regiões apresentam uma proporção mais equilibrada entre escolas gratuitas e não gratuitas.
A Tabela 2 exibe a $\mathrm{CH}$ total do curso e a $\mathrm{CH}$ de SC antes do internato, bem como a porcentagem desta em relação à $\mathrm{CH}$ do curso por região geográfica, administração e gratuidade. Como pode ser observado, não houve diferença na $\mathrm{CH}$ de SC antes do internato, nem em sua porcentagem em relação à $\mathrm{CH}$ do curso por região geográfica, porém, ambas foram maiores em escolas não gratuitas. A média da $\mathrm{CH}$ de $\mathrm{SC}$ antes do internato foi de $487,0$ horas ( $D P=249,3)$ e sua porcentagem em relação à $\mathrm{CH}$ do curso foi de $5,9 \%(D P=3,0)$.

A Tabela 3 exibe as $\mathrm{CH}$ s de teoria e de prática de $\mathrm{SC}$ antes do internato das escolas que forneciam estes dados e a $\mathrm{CH}$ total de SC das escolas que só forneciam a $\mathrm{CH}$ de SC sem discriminar a CH de teoria e de prática, por região geográfica, administração e gratuidade. Como pode ser observado, na Região Norte, a $\mathrm{CH}$ teórica de SC antes do internato foi maior entre nove das 20 escolas que forneciam este dado. Entre as 124 escolas que não discriminavam se a carga era teórica e/ou prática, a $\mathrm{CH}$ de SC antes do internato foi maior naquelas que não eram gratuitas.

Tabela 1. Distribuição de todas as escolas médicas reconhecidas pelo MEC e ativas até 31 de dezembro de 2017, por inclusão no estudo, região, administração e gratuidade.

\begin{tabular}{|c|c|c|c|c|c|c|c|c|c|c|}
\hline \multicolumn{4}{|c|}{ Características da escola } & \multicolumn{4}{|c|}{ Administração (A) } & \multicolumn{3}{|c|}{ Gratuidade (G) } \\
\hline Região geográfica & & Incl & & Privada & Estadual & Municipal & Federal & Sim & Não & Total \\
\hline \multirow{12}{*}{ Norte $^{a, b}$} & \multirow{12}{*}{ I } & \multirow{4}{*}{ Sim } & $\mathrm{n}$ & 8 & 4 & 1 & 7 & 11 & 9 & 20 \\
\hline & & & $(\% \mathrm{I})$ & $(40,0)$ & $(20,0)$ & $(5,0)$ & $(35,0)$ & $(55,0)$ & $(45,0)$ & $(100,0)$ \\
\hline & & & $(\% \mathrm{~A}$ e $\mathrm{G})$ & $(66,7)$ & $(100,0)$ & $(100,0)$ & $(77,8)$ & $(84,6)$ & $(69,2)$ & $(76,9)$ \\
\hline & & & (\%Total) & $(30,8)$ & $(15,4)$ & $(3,8)$ & $(26,9)$ & $(42,3)$ & $(34,6)$ & $(76,9)$ \\
\hline & & \multirow{4}{*}{ Não } & $\mathrm{n}$ & 4 & - & - & 2 & 2 & 4 & 6 \\
\hline & & & $(\% \mathrm{I})$ & $(66,7)$ & - & - & $(33,3)$ & $(33,3)$ & $(66,7)$ & $(100,0)$ \\
\hline & & & $(\% \mathrm{~A}$ e G) & $(33,3)$ & - & - & $(22,2)$ & $(15,4)$ & $(30,8)$ & $(23,1)$ \\
\hline & & & (\%Total) & $(15,4)$ & - & - & $(7,7)$ & $(7,7)$ & $(15,4)$ & $(23,1)$ \\
\hline & & \multirow{4}{*}{ Total } & $\mathrm{n}$ & 12 & 4 & 1 & 9 & 13 & 13 & 26 \\
\hline & & & $(\% \mathrm{I})$ & $(46,2)$ & $(15,4)$ & $(3,8)$ & $(34,6)$ & $(50,0)$ & $(50,0)$ & $(100,0)$ \\
\hline & & & $(\% \mathrm{~A}$ e G) & $(100,0)$ & $(100,0)$ & $(100,0)$ & $(100,0)$ & $(100,0)$ & $(100,0)$ & $(100,0)$ \\
\hline & & & (\%Total) & $(46,2)$ & $(15,4)$ & $(3,8)$ & $(34,6)$ & $(50,0)$ & $(50,0)$ & $(100,0)$ \\
\hline \multirow{12}{*}{ Nordeste $e^{c, d}$} & \multirow{12}{*}{ I } & \multirow{4}{*}{ Sim } & $n$ & 24 & 6 & - & 16 & 22 & 24 & 46 \\
\hline & & & $(\% \mathrm{I})$ & $(52,2)$ & $(13,0)$ & - & $(34,8)$ & $(47,8)$ & $(52,2)$ & $(100,0)$ \\
\hline & & & $(\% A$ e $G)$ & $(66,7)$ & $(46,2)$ & - & $(57,1)$ & $(53,7)$ & $(66,7)$ & $(59,7)$ \\
\hline & & & (\%Total) & $(31,2)$ & $(7,8)$ & - & $(20,8)$ & $(28,6)$ & $(31,2)$ & $(59,7)$ \\
\hline & & \multirow{4}{*}{ Não } & $\mathrm{n}$ & 12 & 7 & - & 12 & 19 & 12 & 31 \\
\hline & & & $(\% \mathrm{I})$ & $(38,7)$ & $(22,6)$ & - & $(38,7)$ & $(61,3)$ & $(38,7)$ & $(100,0)$ \\
\hline & & & $(\% \mathrm{~A}$ e G) & $(33,3)$ & $(53,8)$ & - & $(42,9)$ & $(46,3)$ & $(33,3)$ & $(40,3)$ \\
\hline & & & (\%Total) & $(15,6)$ & $(9,1)$ & - & $(15,6)$ & $(24,7)$ & $(15,6)$ & $(40,3)$ \\
\hline & & \multirow{4}{*}{ Total } & $\mathrm{n}$ & 36 & 13 & - & 28 & 41 & 36 & 77 \\
\hline & & & $(\% \mathrm{I})$ & $(46,8)$ & $(16,9)$ & - & $(36,4)$ & $(53,2)$ & $(46,8)$ & $(100,0)$ \\
\hline & & & $(\% \mathrm{~A}$ e $\mathrm{G})$ & $(100,0)$ & $(100,0)$ & - & $(100,0)$ & $(100,0)$ & $(100,0)$ & $(100,0)$ \\
\hline & & & (\%Total) & $(46,8)$ & $(16,9)$ & - & $(36,4)$ & $(53,2)$ & $(46,8)$ & $(100,0)$ \\
\hline
\end{tabular}


Table 1. (Continuação) Distribuição de todas as escolas médicas reconhecidas pelo MEC e ativas até 31 de dezembro de 2017 , por inclusão no estudo, região, administração e gratuidade.

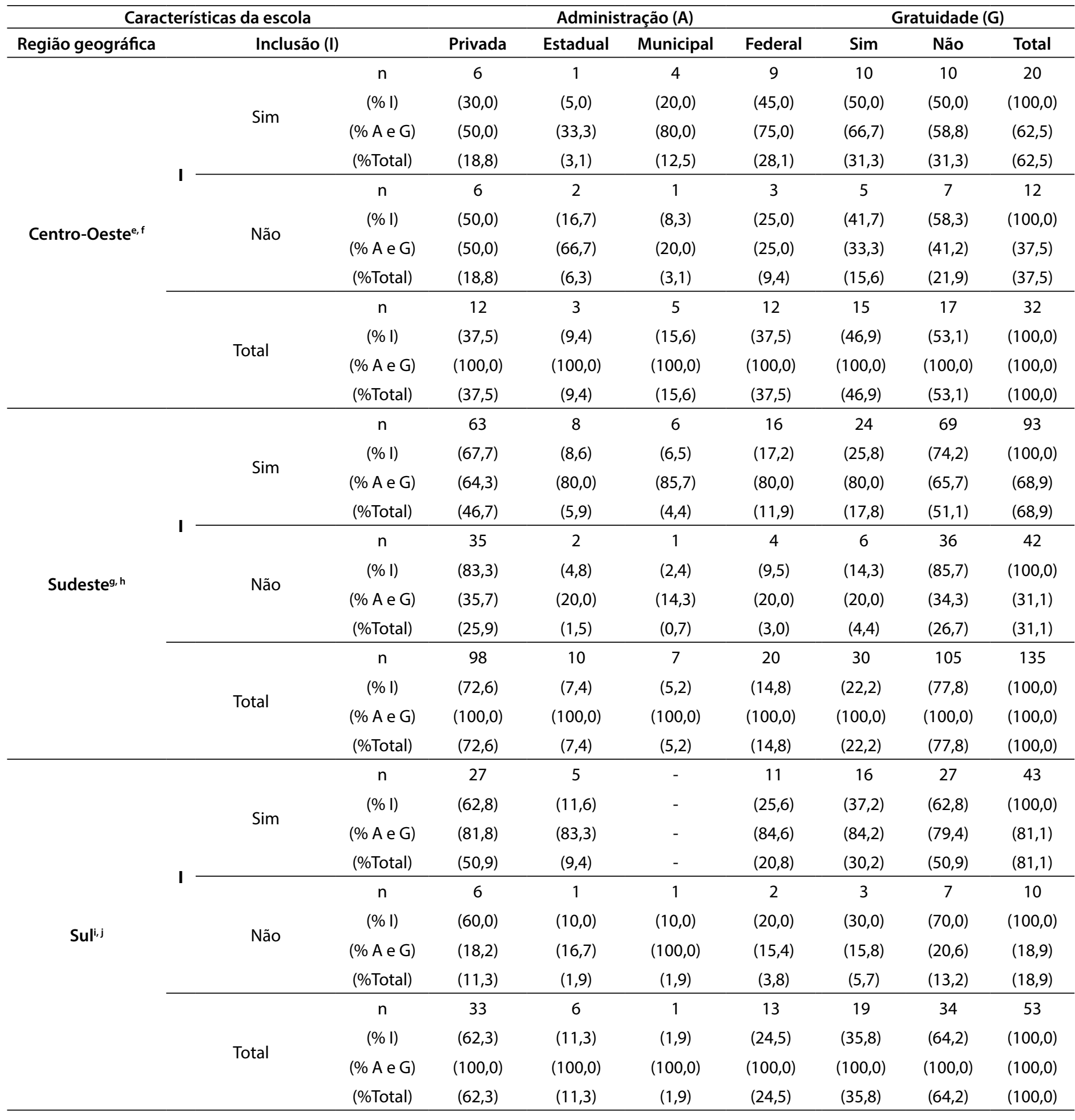


Table 1. (Continuação) Distribuição de todas as escolas médicas reconhecidas pelo MEC e ativas até 31 de dezembro de 2017, por inclusão no estudo, região, administração e gratuidade.

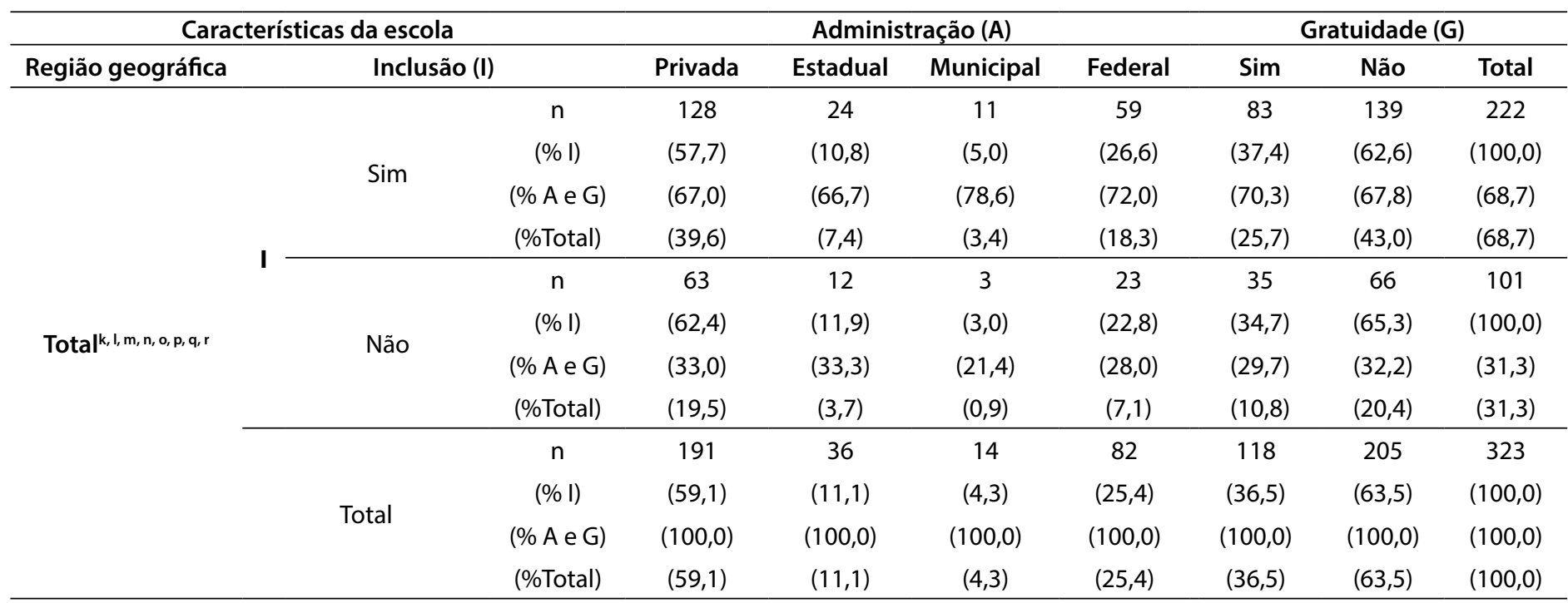

Abreviaturas - MEC: Ministério da Educação; I - inclusão; A - administração; G - gratuidade.

Observação: Análises realizadas com X2 de Pearson.

a Região Norte por inclusão e administração: $X^{2}(3)=2,2, p=0,53$ (6 células com contagem abaixo de 5).

${ }^{b}$ Região Norte por inclusão e gratuidade: $X^{2}(1)=0,87, p=0,352$ (2 células com contagem abaixo de 5).

' Região Nordeste por inclusão e administração: $X^{2}(2)=1,79, p=0,41$.

d Região Nordeste por inclusão e gratuidade: $X^{2}(1)=1,35, p=0,246$.

e Região Centro-Oeste por inclusão e administração: $X^{2}(3)=3,34, p=0,34$ (6 células com contagem abaixo de 5).

${ }^{f}$ Região Centro-Oeste por inclusão e gratuidade: $X^{2}(1)=0,21, p=-0,647$.

${ }^{9}$ Região Sudeste por inclusão e administração: $X^{2}(3)=3,62, p=0,305$ (6 células com contagem abaixo de 5).

${ }^{\mathrm{h}}$ Região Sudeste por inclusão e gratuidade: $X^{2}(1)=2,22, p=0,136$.

'Região Sul por inclusão e administração: $X^{2}(3)=4,43, p=0,218$ (6 células com contagem abaixo de 5).

jRegião Sul por inclusão e gratuidade: $X^{2}(1)=0,18, p=0,669$.

${ }^{k}$ Inclusão por região: $X^{2}(4)=8,08, p=0,89$.

'Inclusão por administração: $X^{2}(3)=1,36, p=0,715$.

m Inclusão por gratuidade: $X^{2}(1)=0,224, p=0,636$.

${ }^{n}$ Número de escolas incluídas e não incluídas: $X^{2}(1)=45,328, p=0,000$.

${ }^{\circ}$ Administração por região em todas as escolas: $X^{2}(12)=40,03, p=0,000$.

${ }^{\mathrm{P}}$ Gratuidade por região em todas as escolas: $X^{2}(4)=24,72, p=0,000$.

${ }^{a}$ Administração por região nas incluídas: $X^{2}(12)=29,97, p=0,03$; e nas não incluídas: $X^{2}(12)=22,84, p=0,029$.

rGratuidade por região nas incluídas: $X^{2}(4)=11,48, p=0,022$; e nas não incluídas: $X^{2}(4)=17,77, p=0,001$.

Fonte: Elaborada pelos autores. 
Tabela 2. Carga horária total do curso e de Saúde Coletiva antes do internato e porcentagem desta em relação à carga horária total do curso das escolas médicas incluídas, reconhecidas pelo MEC e ativas até 31 de dezembro de 2017, por região geográfica, administração e gratuidade.

\begin{tabular}{|c|c|c|c|}
\hline \multirow[b]{2}{*}{ Características da escola } & \multicolumn{3}{|c|}{ Carga horária } \\
\hline & $\begin{array}{c}\text { Curso } \\
\text { Mediana }\left(\mathrm{P}_{25-75}\right)\end{array}$ & $\begin{array}{l}\text { SC antes do internato } \\
\text { Mediana }\left(P_{25-75}\right)\end{array}$ & $\begin{array}{c}\% \text { SC antes do internato/curso } \\
\text { Mediana }\left(P_{25-75}\right)\end{array}$ \\
\hline \multicolumn{4}{|l|}{ Região $0^{a}, b, c$} \\
\hline Norte $(n=20)$ & $8.535,0(7.815,0-8.802,5)$ & $505,0(375,0-720,0)$ & $6,0(4,4-8,6)$ \\
\hline Nordeste $(n=46)$ & $7.980,0(7.564,5-8.721,5)$ & $420,0(276,7-657,00)$ & $4,9(3,3-8,6)$ \\
\hline Centro-Oeste $(n=20)$ & $7.940,0(7.381,5-8.912,0)$ & $393,0(337,5-628,0)$ & $5,1(3,8-8,2)$ \\
\hline Sudeste $(n=93)$ & $8.375,0(7.673,5-8.910,0)$ & $450,0(294,5-674,0)$ & $5,4(3,3-8,1)$ \\
\hline Sul $(n=43)$ & $8.205,0(7.673,0-8.704,0)$ & $420,0(300,0-576,0)$ & $5,3(3,5-7,5)$ \\
\hline \multicolumn{4}{|l|}{ Administração ${ }^{d, e, f}$} \\
\hline Privada $(n=128)$ & $8.276,0(7.631,0-8.880,7)$ & $480,0(330,7-679,2)$ & $6,0(3,9-8,3)$ \\
\hline Municipal $(n=11)$ & $8.500,0(7.607,0-8.963,0)$ & $576,0(360,0-766,0)$ & $6,8(3,7-10,0)$ \\
\hline Estadual $(n=24)$ & $8.527,5(7.857,0-9.669,2)$ & $337,0(281,2-524,2)$ & $4,0(2,6-6,3)$ \\
\hline Federal $(n=59)$ & $8.130,0(7.560,0-8.765,0)$ & $370,0(300,0-480,0)$ & $4,6(3,4-5,9)$ \\
\hline \multicolumn{4}{|l|}{ Gratuidade ${ }^{g, h, i}$} \\
\hline $\operatorname{Sim}(n=83)$ & $8.205,0(7.660,0-8.785,0)$ & $364,0(285,0-504,0)$ & $4,5(3,4-6,2)$ \\
\hline Não $(n=139)$ & $8.280,0(7.607,0-8.903,0)$ & $500,0(336,0-690,0)$ & $6,1(3,9-8,5)$ \\
\hline Total $(n=222)$ & $8.262,5(7.641,7-8.848,5)$ & $440,0(300,0-640,0)$ & $5,4(3,5-7,8)$ \\
\hline
\end{tabular}

Abreviaturas - MEC: Ministério da Educação; SC: Saúde Coletiva.

${ }^{a}$ Carga horária do curso por região: $\mathrm{X}^{2}(4)=3,50, \mathrm{p}=0,48$ (Kruskal-Wallis).

${ }^{\mathrm{b}}$ Carga horária de SC antes do internato por região: $\mathrm{X}^{2}(4)=2,95, \mathrm{p}=0,56$ (Kruskal-Wallis).

c Porcentagem de SC antes do internato em relação ao curso por região: $X^{2}(4)=2,06, p=0,72$ (Kruskal-Wallis).

${ }^{\mathrm{d}}$ Carga horária do curso por administração: $\mathrm{X}^{2}(3)=4,34, \mathrm{p}=0,23$ (Kruskal-Wallis).

e Carga horária de SC antes do internato por administração: $X^{2}(3)=11,48, p=0,009$ (Kruskal-Wallis).

f Porcentagem de SC antes do internato em relação ao curso por administração: $X^{2}(3)=11,52, p=0,009$ (Kruskal-Wallis).

${ }^{9}$ Carga horária do curso por gratuidade: Mann-Whitney $U(U)=5.600,0 . z=-0,364, p=0,716$.

${ }^{\mathrm{h}}$ Carga horária de SC antes do internato por gratuidade: $\mathrm{U}=4.259,0, z=-3,26, p=0,001$.

'Porcentagem de SC antes do internato em relação ao curso por gratuidade: $U=4.283,5, z=--3,21, p=0,001$.

Fonte: Elaborada pelos autores. 
Tabela 3. Carga horária teórica e prática de Saúde Coletiva antes do internato das escolas médicas incluídas, reconhecidas pelo MEC e ativas até 31 de dezembro de 2017, por região, administração e gratuidade.

\begin{tabular}{|c|c|c|c|c|c|c|}
\hline \multirow[b]{3}{*}{ Características da escola } & \multicolumn{6}{|c|}{ Cargas horárias de Saúde Coletiva } \\
\hline & \multicolumn{2}{|r|}{ Teórica ${ }^{a, b, c}$} & \multicolumn{2}{|r|}{ Prática ${ }^{\mathrm{d}, \mathrm{e}, \mathrm{f}}$} & \multicolumn{2}{|c|}{ Não discriminadag, ${ }^{g, i}$} \\
\hline & $\mathbf{n}$ & Mediana $\left(P_{25-75}\right)$ & $\mathbf{n}$ & Mediana $\left(P_{25-75}\right)$ & $\mathbf{n}$ & Mediana $\left(P_{25-75}\right)$ \\
\hline \multicolumn{7}{|l|}{ Região } \\
\hline Norte & 9 & $320,0(257,50-440,0)$ & 9 & $280,0(105,0-470,0)$ & 11 & $440,0(300,0-760,0)$ \\
\hline Nordeste & 16 & $202,5(170,2-307,5)$ & 16 & $194,5(118,7-316,0)$ & 30 & $420,0(247,5-657,0)$ \\
\hline Centro-Oeste & 6 & $190,0(123,5-243,0)$ & 6 & $331,0(130,5-635,0)$ & 14 & $384,0(311,5-592,0)$ \\
\hline Sudeste & 49 & $220,0(171,0-280,0)$ & 48 & $153,5(60,0-330,7)$ & 44 & $470,0(334,5-747,0)$ \\
\hline Sul & 18 & $165,0(119,0-259,5)$ & 18 & $191,0(90,0-327,0)$ & 25 & $420,0(294,0-652,0)$ \\
\hline \multicolumn{7}{|l|}{ Administração } \\
\hline Privada & 49 & $220,0(168,5-330,0)$ & 48 & $237,0(85,5-430,5)$ & 79 & $480,0(340,0-690,0)$ \\
\hline Estadual & 10 & $197,5(143,7-300,0)$ & 10 & $148,5(80,7-283,2)$ & 14 & $300,0(277,5-670,0)$ \\
\hline Municipal & 4 & $98,0(80,7-317,0)$ & 4 & $392,5(52,5-667,2)$ & 7 & $576,0(360,0-766,0)$ \\
\hline Federal & 35 & $225,0(160,0-270,0)$ & 35 & $165,0(90,0-270,0)$ & 24 & $354,0(255,0-565,5)$ \\
\hline \multicolumn{7}{|l|}{ Gratuidade } \\
\hline Sim & 45 & $220,0(153,5-270,0)$ & 45 & $160,0(96,0-267,5)$ & 38 & $337,5(270,0-595,5)$ \\
\hline Não & 53 & $217,0(162,5-330,0)$ & 53 & $237,0(85,5-446,5)$ & 86 & $480,0(360,0-690,0)$ \\
\hline Total & 98 & $218,5(159,0-278,0)$ & 98 & $180,0(90,0-346,5)$ & 124 & $436,5(300,0-663,7)$ \\
\hline
\end{tabular}

Abreviaturas - MEC: Ministério da Educação; n: número de escolas em frequência absoluta; $P_{25-75}$ : percentis 25 e 75.

a Por região: $X^{2}(4)=10,22, p=0,037$ (Kruskal-Wallis).

b Por administração: $X^{2}(3)=3,82, p=0,28$ (Kruskal-Wallis).

cPor gratuidade: Mann-Whitney $U(U)=1.970,0, z=-0,68, p=0,496$.

d Por região: $X^{2}(4)=2,73, p=0,604$ (Kruskal-Wallis).

e Por administração: $X^{2}(3)=1,71, p=0,635$ (Kruskal-Wallis).

f Por gratuidade: $U=1.005,0, z=-1,194, p=0,233$.

${ }^{g}$ Por região: $X^{2}(4)=2,74, p=0,603$ (Kruskal-Wallis).

h Por administração: $X^{2}(3)=8,12, p=0,044$ (Kruskal-Wallis).

'Por gratuidade: $U=1.129 .0, z=-2,738, p=0,006$.

Fonte: Elaborada pelos autores. 
Tabela 4. Carga horária de epidemiologia e estatística e de saúde do trabalhador antes do internato (incluídas no cálculo da carga total de saúde coletiva) por região, administração e gratuidade nas escolas médicas incluídas reconhecidas pelo MEC e ativas até 31 de dezembro de 2017.

\begin{tabular}{|c|c|c|c|c|}
\hline \multirow[b]{3}{*}{ Características da escola } & \multicolumn{2}{|c|}{ Epidemiologia e estatística ${ }^{a, b, c}$} & \multicolumn{2}{|c|}{ Saúde do trabalhador $d, e, f$} \\
\hline & \multicolumn{4}{|c|}{ Carga horária } \\
\hline & $\mathbf{n}$ & Mediana $\left(P_{25-75}\right)$ & $\mathbf{n}$ & Mediana $\left(\mathrm{P}_{25-75}\right)$ \\
\hline \multicolumn{5}{|l|}{ Região } \\
\hline Norte & 13 & $105,0(70,0-162,5)$ & 5 & $60,0(35,0-70,0)$ \\
\hline Nordeste & 28 & $90,0(60,0-120,0)$ & 14 & $45,0(38,5-60,0)$ \\
\hline Centro-Oeste & 10 & $76,0(69,0-93,7)$ & 2 & $67,5(60,0-75,0)$ \\
\hline Sudeste & 65 & $80,0(60,0-120,0)$ & 30 & $40,0(32,2-72,0)$ \\
\hline Sul & 26 & $102,5(68,0-120,0)$ & 12 & $34,5(30,0-58,5)$ \\
\hline \multicolumn{5}{|l|}{ Administração } \\
\hline Privada & 77 & $80,0(60,0-120,0)$ & 37 & $40,0(34,5-66,0)$ \\
\hline Estadual & 17 & $90,0(71,5-143,0)$ & 2 & $45,0(30,0-60,0)$ \\
\hline Municipal & 5 & $67,0(55,0-72,0)$ & 2 & $31,5(30,0-33,0)$ \\
\hline Federal & 43 & $90,0(72,0-120,0)$ & 22 & $47,5(33,0-60,0)$ \\
\hline \multicolumn{5}{|l|}{ Gratuidade } \\
\hline Sim & 60 & $90,0(72,0-120,0)$ & 24 & $47,5(31,0-60,0)$ \\
\hline Não & 82 & $80,0(60,0-120,0)$ & 39 & $40,0(33,0-60,0)$ \\
\hline Total & 124 & $88,0(60,0-120,0)$ & 63 & $40,0(33,0-60,0)$ \\
\hline
\end{tabular}

Abreviaturas - MEC: Ministério da Educação; n: número de escolas em frequência absoluta; $\mathrm{P}_{25-75}$ : percentis 25 e 75.

aPor região: $X^{2}(4)=6,63, p=0,157$ (Kruskal-Wallis).

bPor administração: $\mathrm{X}^{2}(3)=5,05, \mathrm{p}=0,168$ (Kruskal-Wallis).

c Por gratuidade: Mann-Whitney U $(U)=2.060,0, z=-1,657, p=0,098$.

d Por região: $X^{2}(4)=5,25, p=0,263$ (Kruskal-Wallis).

e Por administração: $X^{2}(3)=2,58, p=0,46$ (Kruskal-Wallis).

${ }^{f}$ Por gratuidade: $\mathrm{U}=448,0, z=-0,286, p=0,775$.

Fonte: Elaborada pelos autores.

A Tabela 4 exibe as $\mathrm{CHs}$ de EB e de ST por região geográfica, administração e gratuidade. Não houve diferença em nenhuma das variáveis estudadas.

\section{DISCUSSÃO}

Nosso estudo incluiu 68,7\% das EM reconhecidas pelo MEC e ativas até 31 de dezembro de 2017. Constatamos um maior número de escolas não gratuitas e privadas no Brasil, especialmente na Região Sudeste. Também verificamos que há maior proporção de escolas privadas e não gratuitas nas Regiões Sul e Sudeste e uma distribuição mais equilibrada entre escolas gratuitas e não gratuitas nas outras regiões, a qual poderia ser parcialmente. Esse maior equilíbrio, talvez, possa em parte justificado pela expansão das EM federais, de 2013 a 2015, decorrente do Programa Mais Médicos, implementado em $2013^{20}$. Nesse período, foram criadas 30 escolas: 13 no Nordeste, três no Norte, quatro no Centro-Oeste, cinco no Sudeste e cinco no Sul${ }^{20}$.
Outro achado foi uma $\mathrm{CH}$ de SC antes do internato maior entre as escolas não gratuitas, porém, não conseguimos aventar uma hipótese plausível para esse achado.

Ainda, a CH teórica de SC antes do internato foi maior na Região Norte. Entretanto, cabe ressaltar que apenas nove escolas dessa região disponibilizavam suas $\mathrm{CH}$ s teórica e prática de forma discriminada, o que pode ter levado a um viés nessa análise, pois, ao considerarmos a $\mathrm{CH}$ total de $\mathrm{SC}$, não houve diferença entre as regiões.

$\mathrm{Na}$ literatura, identificamos artigos nacionais em forma de estudo ou relato de caso, entre eles um realizado em três universidades do Paraná ${ }^{21}$, um sobre a Universidade Estadual de Londrina (UEL) e seu processo de transformação curricular ${ }^{22}$ e outro sobre o currículo da Faculdade de Medicina de Marília (Famema) ${ }^{23}$. Há também estudos sobre a Universidade Estadual do Ceará (Uece) ${ }^{18,24}$. Porém, devido à inexistência de recomendações de limites de $\mathrm{CH}$ para SC antes do internato nas DCN e de estudos nacionais abrangentes que contemplassem 
a maioria das escolas brasileiras, não foi possível compararmos nossos achados com dados nacionais. Essa lacuna foi a principal motivação para realização de nosso estudo. Encontramos, porém, recomendações e estudos internacionais que possibilitaram comparações.

O Ministério da Educação de Cuba preconiza que o currículo do curso de Medicina tenha $\mathrm{CH}$ de 1.242 horas antes do internato destinadas às disciplinas analisadas em nosso estudo ${ }^{25,26}$. Esse valor equivale a mais que o dobro do percentil $75\left(P_{75}\right)$ obtido em nosso estudo.

No curso de Medicina da Universidade de Toronto, no Canadá, o período antes do internato é de dois anos ${ }^{27}$, no qual a $\mathrm{CH}$ de SC é de 328 horas $^{28}$. Esse valor é próximo ao percentil $25\left(\mathrm{P}_{25}\right)$ de nosso estudo. Revendo a evolução do currículo dessa universidade ao longo de dez anos, constatouse que a redistribuição da $\mathrm{CH}$ de $\mathrm{SC}$ ao longo dos dois anos antes do internato, sem alterar a $\mathrm{CH}$ total, resultou em maior aproveitamento dos alunos ${ }^{28}$.

Alguns outros estudos internacionais citam a $\mathrm{CH}$, mas não especificam se ela se refere ao total do curso ou apenas ao período antes do internato. Um estudo catalão ${ }^{29}$, com quatro $E M$, encontrou uma variação de 1,5 a 12 créditos nas $\mathrm{CHs}$. No Sistema Europeu de Transferências e Acumulação de Créditos, cada crédito equivale de 25 a 30 horas $^{30}$. Assim, a CH de SC do estudo catalão variou de 37,5 a 360 horas $^{29}$, valor abaixo do $P_{25}$ encontrado em nosso estudo.

Um estudo com 16 EM de seis países do Leste Europeu encontrou uma variação de 235 a 615,0 horas na $\mathrm{CH}$ de $\mathrm{SC}^{31}$. Outro estudo com 32 EM de 18 países da Europa constatou uma variação de 18,5 a 500 horas nas $\mathrm{CHs}^{32}$. Ainda que não fosse possível saber se a $\mathrm{CH}$ incluía o internato, entre as $16 \mathrm{EM}$ do estudo do Leste Europeu ${ }^{31}$, em 13 a CH de SC estava entre o $P_{25}$ e $\mathrm{P}_{75}$ de nosso estudo e, em duas, abaixo do $\mathrm{P}_{25}$ de nosso estudo. Quanto ao estudo com 32 escolas europeias ${ }^{32}$, em dez a $\mathrm{CH}$ de SC ficava entre o $P_{25}$ e $P_{75}$ de nosso estudo e, em 22, abaixo do $P_{25}$.

Na Grã-Bretanha, em 2008, entre 29 de 31 escolas, a média da porcentagem do ensino de SC em relação ao total do curso era de $13,0 \%$, variando de 3,4 a 20,0\%. Os autores não especificaram se a CH de SC era relativa ao período antes do internato ou de todo o curso ${ }^{33}$. Essa média fica bem acima da mediana encontrada em nosso estudo. Porém, ressaltamos que essa porcentagem pode representar a $\mathrm{CH}$ de $\mathrm{SC}$ de todo o curso, incluindo o internato.

Quanto à CH de ST, em um estudo na Grã-Bretanha ${ }^{34}$, o conteúdo variou de zero a mais de seis horas semanais, enquanto, em outro no México ${ }^{35}$ com $35 \mathrm{EM}$, a disciplina de ST durava um semestre e a $\mathrm{CH}$ variou de uma a oito horas semanais. No estudo com 16 escolas do Leste Europeu ${ }^{31}$, a CH de ST variou de 30 a 165 horas. Ao comparar a $\mathrm{CH}$ dessas escolas com a encontrada em nosso estudo, constatamos que ela estava abaixo do $P_{25}$ em uma escola, acima do $P_{75}$ em dez e entre o $P_{25}$ e $\mathrm{P}_{75}$ em quatro. No estudo com 32 escolas europeias ${ }^{32}$, a $\mathrm{CH}$ variou de zero a 105 horas, ficando cinco delas abaixo do $P_{25}$ de nosso estudo, quatro acima do $P_{75}$ e três entre o $P_{25}$ e $P_{75}$.

Quanto à $\mathrm{CH}$ de EB, em Cuba ${ }^{26}$ ela era de 120 horas, o que equivale ao $P_{75}$ de nosso estudo. No Leste Europeu ${ }^{31}$, essa $\mathrm{CH}$ variou de 75 a 180 horas, ficando a $\mathrm{CH}$ de quatro EM acima do $P_{75}$ de nosso estudo e as 11 restantes entre o $P_{25}$ e $P_{75}$. No estudo com 32 escolas europeias ${ }^{32}$, a CH foi de 7,0 a 225 horas, ficando a $\mathrm{CH}$ de 11 escolas abaixo do $\mathrm{P}_{25}$ de nosso estudo, de cinco acima do $P_{75}$ e de sete entre o $P_{25}$ e $P_{75}$.

As limitações de nosso estudo incluem a impossibilidade de diferenciar a $\mathrm{CH}$ teórica e a $\mathrm{CH}$ prática em quase metade das escolas incluídas, bem como de incluir escolas que não explicitavam a $\mathrm{CH}$ destinada à $\mathrm{SC}$ em seus módulos integrados. Ainda que a integração curricular seja preconizada, idealmente a $\mathrm{CH}$ destinada a cada área poderia ser especificada a fim de fornecer parâmetros objetivos de comparação. Outra limitação foi a análise de dados disponíveis apenas na internet.

Para maior compreensão sobre o ensino de SC, sugerimos estudos futuros que analisem a concepção política e pedagógica e a corrente de pensamento que fundamenta o ensino de SC das escolas, a distribuição de SC ao longo do curso, seus conteúdos e estratégias e cenários de ensinoaprendizagem, incluindo a avaliação do estudante. Para tanto, seria necessário fazer uma análise pormenorizada dos projetos políticos do curso e dos planos de ensino-aprendizagem, associada a entrevistas com gestores, docentes e discentes.

\section{CONCLUSÕES}

As escolas incluídas tiveram representatividade quanto à administração e gratuidade em cada região geográfica.

Houve grande variabilidade na mediana de $\mathrm{CH}$ de SC antes do internato, bem como na de sua porcentagem em relação à $\mathrm{CH}$ total, e essas medianas foram maiores em escolas não gratuitas, as quais têm maior proporção nas regiões Sudeste e Sul.

Os conteúdos de EB e ST são similares por região, administração e gratuidade.

\section{CONTRIBUIÇÃO DOS AUTORES}

Sofia Romay Oliveira participou na elaboração do projeto, na coleta e análise de dados e na redação do manuscrito, que foi seu trabalho de conclusão de curso, requisito do curso de graduação em Medicina da UFSC. Athos Paulo Santos Martini participou na elaboração do projeto, na coleta e análise de dados e na redação e revisão do manuscrito. Suely Grosseman participou na orientação e elaboração do projeto, na coleta e análise de dados, e na redação e revisão do manuscrito. 


\section{CONFLITO DE INTERESSES}

Declaramos não haver conflito de interesses neste estudo.

\section{FINANCIAMENTO}

\section{Declaramos que não houve financiamento neste estudo.}

\section{REFERÊNCIAS}

1. Horton R. A new epoch for health professionals' education. Lancet. 2010;376(9756):1875-7.

2. Tambellini AT, Câmara VM. A temática saúde e ambiente no processo de desenvolvimento do campo da saúde coletiva: aspectos históricos, conceituais e metodológicos. Cien Saude Colet 1998;3(2):47-59.

3. Grosseman S, Patrício ZM. Do desejo à realidade de ser médico. Florianópolis: Editora da UFSC; 2004.

4. Almeida MJ. A educaçäo médica e as atuais propostas de mudança: alguns antecedentes históricos. Rev Bras Educ Med. 2001;25(2):42-52 [acesso em 24 out 2018]. Disponível em: http://www.educacaomedica. org.br/UserFiles/File/2001/Volume25 2/educacao_medica.pdf.

5. Gibbon W. Medical schools for the health-care needs of the 21 st century. Lancet. 2007;369(9580):2211-3.

6. Johnson I, Donovan D, Parboosingh J. Steps to improve the teaching of public health to undergraduate medical students in Canada. Acad Med. 2008;83(4):414-8.

7. Tyler IV., Hau M, Buxton JA, Elliott LJ, Harvey BJ, Hockin JC, et al. Canadian medical students' perceptions of public health education in the undergraduate medical curriculum. Acad Med. 2009;84(9):1307-12.

8. Jin H, Dong G, Zou L, Shen X, Li D. History and status quo of higher public health education in China. Public Health Rev. 2020;41:1-16.

9. Luque-Fernández MA, Calduch EN. Education in public health, epidemiology and biostatistics in Spain from a global and comparative perspective. Gac Sanit. 2019;33(6):502-3.

10. Bell C, Simmons A, Martin E, McKenzie C, McLeod J, McCoombe S. Competent with patients and populations: Integrating public health into a medical program. BMC Med Educ. 2019;19(1):1-9.

11. Gillam S, Rodrigues V, Myles P. Public health education in UK medical schools: towards consensus. J Public Healh. 2016;38(3):522-5.

12. Nagesh NM, Giurca BC. Empowering undergraduate students to pioneer public health education: a call for action. Perspect Public Health. 2019;139(4):179-80.

13. Ofori-Adjei D. Changing landscape of public health and medical education curriculum. Ghana Med J. 2018;52(3):115-115.

14. Brasil. Resolução CNE/CES no 4, de 7 de novembro de 2001. Institui Diretrizes Curriculares Nacionais do Curso de Graduação em Medicina. Diário Oficial da União; 9 nov 2001. Seção 1, p. 38.

15. Brasil. Resolução CNE/CES n 3, de 20 de junho de 2014. Institui Diretrizes Curriculares Nacionais do Curso de Graduação em Medicina. Diário Oficial da União; 23 jun. 2014. Seção 1, p. 8-11.

16. Lima-Gonçalves E. Médicos e ensino da medicina no Brasil. São Paulo: Edusp; 2002.

17. BRASIL. e-MEC: Instituições de educação superior e cursos cadastrados [acesso em 14 ago 2018]. Brasília: Ministério da Educação; 2007. Disponível em: http://emec.mec.gov.br.

18. Oliveira JAA, Jorge MSB, Silva MGC, Pinto DM, Pinto FJM. A saúde coletiva na formação dos discentes do curso de Medicina da Universidade Estadual do Ceará, Brasil. Rev Bras Educ Med. 2011;35(3):398-404.
19. Conselho Federal de Medicina. Resolução $n^{\circ} 1.488 / 98$, de 11 de fevereiro de 1998. Normas específicas para médicos que atendam o trabalhador. Diário Oficial da União; 6 mar 1998. Seção I, p. 150.

20. Cyrino EG, de Sordi MRL, Mendes GSCV, Luna WF, Mendonça CS, Alexandre FLF, et al. Mapeamento das características da implantação de novos cursos de Medicina em universidades federais brasileiras. Rev Panam Salud Publica. 2020 Oct;44:e117 [acesso em 22 out 2020]. Disponível em: https://iris.paho.org/handle/10665.2/52802.

21. de Campos JJB, Elias PEM, Cordoni Junior L. O ensino da saúde coletiva na graduação médica: estudo de caso em três universidades do Paraná. Sao Paulo Med J. 2009;127(6):335-41.

22. de Campos JJB, Elias PEM. Universidade Estadual de Londrina: reflexões iniciais. Rev Bras Educ Med. 2008;32(2):149-59.

23. Feuerwerker LCM. Além do discurso de mudança na educação médica: processos e resultados. São Paulo: Hucitec; 2002.

24. Gomes NN. Avaliação da formação de profissionais médicos de uma universidade pública em Fortaleza-CE [dissertação]. Fortaleza: Universidade Estadual do Ceará; 2014.

25. Valdés JV, Sacasas JF. Las asignaturas de salud pública en las políticas de formación para la carrera de medicina. Rev Cuba Salud Pública. 2012:38(3):383-92 [acesso em 14 jul 2020]. Disponível em: http://scielo. sld.cu/scielo.php?script=sci_arttext\&pid=S0864-34662012000300005.

26. Aguilera EAM, Díaz NT, Sacasas JF, Gómez MP, Figueredo SS, Cobelo JMD. Cronología de los mapas curriculares en la carrera de Medicina. Educ Med Super. 2015;29(1)93-107 [acesso em 16 jul 2020]. Disponível em: $\quad$ http://scielo.sld.cu/scielo.php?script=sci_arttext\&pid=S086421412015000100010\&lng=es.

27. Newman A. The New Toronto Medical Curriculum. Biochem Educ 1993;21(4):170-9 [acesso em 16 jul 2020]. Disponível em: https://www. sciencedirect.com/science/article/pii/030744129390085E.

28. Johnson IL, Scott FE, Byrne NP, MacRury KA, Rosenfield J. Integration of community health teaching in the undergraduate medicine curriculum at the University of Toronto. Am J Prev Med. 2011;41(4 Suppl 3):S176-80. doi: 10.1016/j.amepre.2011.06.003.

29. Obradors-Rial N, Segura-Benedicto A. La salud pública en las facultades de medicina y escuelas de enfermería de Cataluña: la visión del alumnado. Educ Med. 2010;13(2):113-22.

30. European Communities. ECTS Users' Guide. 2009 [acesso em 14 out 2018] Disponível em: http://ec.europa.eu/dgs/education_culture/repository/ education/tools/docs/ects-guide_en.pdf.

31. Likic R, Dusek T, Horvat D. Analysis and prospects for curricular reform of medical schools in Southeast Europe. Med Educ. 2005;39(8):833-40. doi: 10.1111/j.1365-2929.2005.02228.x.

32. DusekT, Bates T. Analysis of European medical schools' teaching programs. Croat Med J. 2003;44(1):26-31 [acesso em 17 out 2018]. Disponível em: http://www.ncbi.nlm.nih.gov/pubmed/12590425.

33. Harding A, Rosenthal J, Al-Seaidy M, Gray DP, McKinley RK. Provision of medical student teaching in UK general practices: a cross-sectional questionnaire study. Br J Gen Pract. 2015; 65(635):e409-17. doi: 10.3399/ bjgp15X685321

34. Williams N, Wynn PA, Whitaker S. Undergraduate occupational medicine tuition in UK schools of medicine. Occup Med (Chic III). 2011;61(3):152-6. doi: 10.1093/occmed/kqr010.

35. Sánchez-Román FR, Medina-Figueroa AM, Rangel-Zertuche RA, SánchezRamos A. Enseñanza de medicina del trabajo en el pregrado de las escuelas de medicina en México. Salud Publica Mex. 2009;51(1):97-103. 